\title{
Molecular epidemiology of a large outbreak of multiresistant Klebsiella pneumoniae
}

\author{
T. M. A. WELLER, F. M. MACKENZIE and K. J. FORBES*
}

Department of Medical Microbiology, Aberdeen Royal Infirmary, Foresterhill, Aberdeen AB25 $2 Z N$ and *Department of Medical Microbiology, Aberdeen University, Foresterhill, Aberdeen AB25 2ZD

\begin{abstract}
An outbreak of multiresistant Klebsiella pneumoniae has continued in the Grampian Region of Scotland since 1992. The organism, which generally produces an extendedspectrum $\beta$-lactamase (ESBL), has spread to several hospitals and nursing homes. DNA from 80 possible outbreak isolates was digested with the restriction endonucleases $X b a I$ and SpeI, and the patterns obtained by pulsed-field gel electrophoresis were compared. Restriction patterns of 79 of the isolates were found to be highly similar with both restriction enzymes, whereas one isolate was unrelated. The outbreak isolates were divided into six subtypes with SpeI and 16 subtypes with XbaI. These subtypes were independent of antibiotic susceptibility pattern, date of isolation and ward of origin, but the XbaI subtype did correlate with the SpeI subtype. It was concluded that the Klebsiella isolates of this outbreak were clonally related.
\end{abstract}

\section{Introduction}

Members of the Enterobacteriaceae commonly cause hospital-acquired outbreaks of infection. These become more serious when the bacteria are resistant to antibiotics, particularly third-generation cephalosporins, quinolones and aminoglycosides. Since 1992 the Grampian region of Scotland has experienced a marked increase in the number of multiresistant Klebsiella pneumoniae isolates. The course of this outbreak has been described in detail elsewhere [1], but briefly, six sporadic cases occurred over a 10-month period, followed by a cluster of seven cases in the intensive care unit (ICU) in 1993. By the end of December 1994 a total of 283 patients had been involved at six hospitals or cottage hospitals within the region and at several nursing homes.

The Klebsiella isolates characteristically expressed an extended-spectrum $\beta$-lactamase (ESBL) active against third-generation cephalosporins. Most were also resistant to gentamicin and ciprofloxacin, although imipenem and amikacin remained effective agents. Some isolates that lacked ESBL activity, but were resistant to gentamicin or ciprofloxacin or both, were also thought to be part of the outbreak.

Outbreaks of ESBL-producing klebsiellae studied

Received 26 Nov. 1996; revised version accepted 22 Feb. 1997.

Corresponding author: Dr T. M. A. Weller. elsewhere have entailed the dissemination of a single clone from patient to patient [2-4], or the transfer of resistance genes among strains [5-7]. Isolates thought to be part of the Grampian outbreak had a diverse range of susceptibility patterns and so their mode of spread of resistance was unclear. Pulsed-field gel electrophoresis (PFGE) has been found to be a useful method for typing many bacterial species including $K$. pneumoniae $[3,8,9]$, and it was used here to assess the relatedness of multiresistant isolates thought to be part of the present outbreak.

\section{Materials and methods}

\section{Bacterial strains}

Isolates of $K$. pneumoniae were identified previously and described by Hobson et al. [1]. A selection of these, from two of the hospitals involved (A and B) were examined in the present study. Hospital A is the major acute hospital for Grampian region, (population 500000 ) situated in Aberdeen. It provides 1467 beds, including the regional ICU. Hospital B is a non-acute facility with 497 beds providing medicine for the elderly, orthopaedics and rehabilitation. The test organisms were collected over the whole period of the outbreak and were from patients on every affected ward. A more detailed analysis was made of the isolates from one ward (ward C) in hospital A by testing 24 of the 47 isolates obtained from patients, and all seven obtained by environmental screening. This group included nine isolates cultured from one patient 
(patient D) at different times over a 4-month period. Eleven locally isolated $K$. pneumoniae that were ESBL-negative and susceptible to gentamicin and ciprofloxacin were included as controls along with strain ATCC 9633.

\section{Susceptibility testing}

Minimum inhibitory concentrations (MICs) of cefotaxime, cefotaxime with clavulanic acid $(2 \mathrm{mg} / \mathrm{L})$, ceftibuten, cefotetan, gentamicin, ciprofloxacin and imipenem were determined by the NCCLS agar dilution method [10] with Mueller-Hinton agar and an inoculum of $10^{4} \mathrm{cfu} / \mathrm{spot}$. The presence of ESBL activity was demonstrated by the Jarlier double-disk diffusion test [11] with co-amoxiclav and cefotaxime disks.

\section{Capsular typing}

The multiresistant isolates and controls were typed by the Quellung test with antiserum to type K2. Those found not to be serotype $\mathrm{K} 2$ by this method were further typed by counterimmuno-electrophoresis at the Central Public Health Laboratory, Colindale, London.

\section{PFGE}

A modification of the method first described by Smith et al. [12] was used. Bacterial isolates were recovered from storage at $-70^{\circ} \mathrm{C}$ and cultured overnight on Columbia Agar (Oxoid) at $37^{\circ} \mathrm{C}$. They were then harvested into $1 \mathrm{ml}$ of TEN buffer $(0.1 \mathrm{M} \mathrm{TrisCl}$, $0.15 \mathrm{M} \mathrm{NaCl}, 0.1 \mathrm{M}$ EDTA), washed twice in $1 \mathrm{ml}$ of the same buffer, then resuspended in $0.3 \mathrm{ml}$ of EC buffer ( $6 \mathrm{mM}$ TrisCl, $1 \mathrm{M} \mathrm{NaCl}, 0.1 \mathrm{M}$ EDTA, 20 cetyl ether $0.5 \%$, deoxycholate $0.2 \%$, N-lauroylsarcosine $0.5 \%)$. A $50-\mu 1$ sample of the suspension was mixed with $200 \mu \mathrm{l}$ of InCert agarose (Flowgen Instruments, Lichfield, Staffs.) $1 \%$ and allowed to set in a gel plug mould. The plug was incubated overnight at $37^{\circ} \mathrm{C}$ in $2 \mathrm{ml}$ of EC buffer containing lysozyme (Boehringer Mannheim, Mannheim, Germany) $1 \mathrm{mg} / \mathrm{ml}$. This buffer was then replaced by $2 \mathrm{ml}$ of PB buffer $(0.5 \mathrm{M}$ EDTA, N-lauroylsarcosine $1 \%$ ) containing proteinase $\mathrm{K}$ (Boehringer Mannheim) $1 \mathrm{mg} / \mathrm{ml}$ and incubated for a further $24 \mathrm{~h}$ at $50^{\circ} \mathrm{C}$. Finally, the plug was washed six times in $5 \mathrm{ml}$ of TE buffer $(20 \mathrm{mM}$ TrisCl, $5 \mathrm{mM}$ EDTA) before storage at $4^{\circ} \mathrm{C}$ in $1 \mathrm{ml}$ of TE buffer until needed.

DNA was digested with $20 \mathrm{U}$ of the restriction endonuclease $\mathrm{XbaI}$ (Advanced Biotechnologies, Epsom, Surrey): a slice of the plug c. $5 \mathrm{~mm} \times 5 \mathrm{~mm}$ was incubated with the enzyme at $37^{\circ} \mathrm{C}$ for $4 \mathrm{~h}$ in $200 \mu \mathrm{l}$ of 10-fold diluted reaction buffer (as supplied with $X b a \dot{\mathrm{I}}$ ), containing $1 \mu 1$ of bovine serum albumin (BSA) $1 \mathrm{mg} / \mathrm{ml}$ and $5 \mu \mathrm{l}$ of $20 \mathrm{mM}$ dithiothreitol (DTT)). DNA fragments were separated in an agarose $1 \%$ gel electrophoresed in $0.5 \times$ TBE on a CHEF-DR III electrophoresis cell (BioRad, Richmond, CA,
USA). The following conditions were used: $6 \mathrm{~V} / \mathrm{cm}$ for $24 \mathrm{~h}$, with the pulse time ramped linearly from $10 \mathrm{~s}$ to $70 \mathrm{~s}$. The gel was stained with ethidium bromide $0.5 \mathrm{mg} / \mathrm{L}$ and examined under ultraviolet light. The banding patterns were analysed visually according to the criteria of Tenover et al. [13].

DNA from 38 isolates, selected to include all the PFGE types produced with $X b a I$, and the ATCC control strain, was also subjected to digestion at $37^{\circ} \mathrm{C}$ for $16 \mathrm{~h}$ with $20 \mathrm{U}$ of restriction endonuclease SpeI (Advanced Biotechnologies) in $200 \mu \mathrm{l}$ of 10-fold diluted restriction enzyme buffer (supplied with the enzyme) and supplemented with $1 \mu \mathrm{l}$ of BSA $1 \mathrm{mg} / \mathrm{ml}, 5 \mu \mathrm{l}$ of $20 \mathrm{mM}$ DTT. The pulse time was ramped linearly from $2 \mathrm{~s}$ to $30 \mathrm{~s}$, but otherwise the running conditions were identical to those for Xbal digestions.

\section{Results}

A total of 92 isolates of $K$. pneumoniae was studied, comprising 11 randomly selected local, non-outbreak isolates, one control strain (ATCC 9633) and 80. multiresistant isolates. The latter isolates comprised 73 from clinical specimens and seven from environmental screening. The isolates from patients originated from 56 individuals, with single isolates from 48 and between two and nine isolates from the other eight. Forty-two isolates were from urine, 13 from stools, 11 from respiratory specimens, four from wounds and one each from blood, a central venous line tip and a hand. Of the resistant isolates, 56 were from hospital $\mathrm{A}$, including 31 from ward C; 22 were from hospital B and two were from general practice. All seven environmental isolates were from ward $C$.

\section{Capsular typing and antimicrobial susceptibility}

Capsular typing revealed that all the multiresistant isolates were of serotype $\mathrm{K} 2$. One control isolate was also typed as $\mathrm{K} 2$ but the remainder were of other capsular types.

Sixty-eight resistant test isolates, but none of the control strains, showed ESBL activity. The 12 test isolates without ESBL activity were susceptible to cefotaxime at $0.12-1 \mathrm{mg} / \mathrm{L}$ whereas MICs for those with the enzyme ranged from $1->64 \mathrm{mg} / \mathrm{L}$ (Table 1). Addition of clavulanic acid $2 \mathrm{mg} / \mathrm{L}$ reduced the MICs of cefotaxime for all the ESBL-positive isolates to $<1 \mathrm{mg} / \mathrm{L}$. MICs of ceftibuten and the cephamycin, cefotetan, were always lower than those of cefotaxime for the ESBL producers. Two test isolates were susceptible to ciprofloxacin (MIC $<1 \mathrm{mg} / \mathrm{L}$ ) and three isolates were susceptible to gentamicin (MIC $<1 \mathrm{mg} / \mathrm{L}$ ); the remainder were resistant. A single isolate exhibited resistance to imipenem (MIC $8 \mathrm{mg} / \mathrm{L}$ ). 
Table 1. Minimum inhibitory concentrations of antibiotics for the test isolates

\begin{tabular}{|c|c|c|c|c|c|c|c|c|c|c|c|c|}
\hline \multirow[b]{2}{*}{ Antimicrobial agent } & \multicolumn{12}{|c|}{ Number of isolates with MIC $(\mathrm{mg} / \mathrm{L})$ of } \\
\hline & 0.03 & 0.06 & 0.12 & 0.25 & 0.5 & 1 & 2 & 4 & 8 & 16 & 32 & $>32$ \\
\hline Cefotaxime & $\ldots$ & ... & 6 & 3 & 2 & 4 & 3 & 14 & 7 & 7 & 20 & 14 \\
\hline Cefotaxime + clav* & $\ldots$ & 5 & 58 & 14 & 3 & & & $\ldots$ & $\ldots$ & $\ldots$ & $\ldots$ & $\ldots$ \\
\hline Ciprofloxacin & i & $\ldots$ & $\ldots$ & 1 & $\ldots$ & 49 & 29 & $\ldots$ & $\ldots$ & $\ldots$ & $\ldots$ & $\ldots$ \\
\hline Gentamicin & $\ldots$ & $\ldots$ & $\ldots$ & 1 & 2 & 1 & 1 & $\ldots$ & 4 & 42 & 23 & 6 \\
\hline Imipenem & $\ldots$ & 1 & 73 & 3 & 2 & & & $\ldots$ & 1 & $\ldots$ & $\ldots$ & $\ldots$ \\
\hline Ceftibuten & 1 & 5 & 8 & 12 & 10 & 12 & 8 & 16 & 6 & $\ldots$ & $\ldots$ & 2 \\
\hline Cefotetan & $\ldots$ & 2 & 9 & 23 & 17 & 17 & 7 & 2 & $\ldots$ & $\ldots$ & $\ldots$ & 3 \\
\hline
\end{tabular}

${ }^{*}$ Clavulanic acid (clav) was used at a fixed concentration of $2 \mathrm{mg} / \mathrm{L}$.

\section{PFGE}

The 80 multiresistant isolates gave 17 distinct PFGE patterns when their DNA was digested with $X b a I$ (Table 2, Fig. 1) The commonest pattern (pattern X1a) was seen for $30(37 \%)$ of the multiresistant isolates. All patterns were compared by the criteria of Tenover et al. [13] which state that patterns with no differences are indistinguishable, those with one-to-three fragment differences are probably related, those with four-to-six differences are possibly related and those with seven or more differences are unrelated. On this basis 30 isolates were indistinguishable from pattern X1a, 37 were probably related, 12 were possibly related, and one was unrelated (pattern X2). All the randomly selected local non-outbreak isolates, and the control, produced patterns that differed from pattern Xla and from each other by at least seven bands. These same overall results were obtained whichever pattern was used as the comparitor.

PFGE patterns for 38 multiresistant isolates were also analysed after digestion with the restriction endonuclease SpeI (Fig. 1). Seven distinct PFGE patterns were obtained, each encompassing between one and six of the groups defined with $X b a \mathrm{I}$ (Table 2). Thus, isolates with minor differences in $X b a I$ PFGE pattern were often indistinguishable with SpeI, but those indistinguishable by $X b a \mathrm{I}$ were never separated by Spe I. Pattern S1a was the commonest pattern and all others were compared to it. Two other patterns were probably related and three possibly related by the criteria of Tenover et al. [13]. The one resistant isolate found to give a unique pattern after digestion with $X b a I$ also produced a pattern (S2) unrelated to pattern Sla.

The groupings produced by PFGE did not correlate with any other variable examined. Thus, the PFGE type was independent of the date of isolation, the ward of origin of isolate, its susceptibility pattern and the presence of an ESBL.

\section{Typing of isolates from ward $C$}

A total of 31 isolates ( 24 from patients and seven from environmental sources) from ward $\mathrm{C}$ were analysed. Fifteen isolates $(48 \%)$ gave the pattern $\mathrm{Xla}$, eight (26\%) gave pattern X1c, the remainder gave patterns X1h (10\%), X1b (6\%), X1d (3\%), X1e (3\%) and X1j

Table 2. Genotypic characteristics of $K$. pneumoniae isolates studied by PFGE

\begin{tabular}{|c|c|c|c|c|c|}
\hline \multicolumn{3}{|c|}{ SpeI } & \multicolumn{3}{|c|}{$X b a \mathrm{I}$} \\
\hline $\begin{array}{l}\text { PFGE } \\
\text { pattern }\end{array}$ & $\begin{array}{c}\text { Number of } \\
\text { isolates }\end{array}$ & $\begin{array}{l}\text { Differences } \\
\text { from } \\
\text { pattern Sla }\end{array}$ & $\begin{array}{c}\text { PFGE } \\
\text { pattern }\end{array}$ & $\begin{array}{l}\text { Number of } \\
\text { isolates }\end{array}$ & $\begin{array}{l}\text { Differences } \\
\text { from } \\
\text { pattern } \times 1 a\end{array}$ \\
\hline \multirow[t]{6}{*}{ Sla } & 22 & - & Xla & 30 & - \\
\hline & & & $\mathrm{X} 1 \mathrm{~b}$ & 11 & 1 \\
\hline & & & $\mathrm{X} 1 \mathrm{c}$ & 12 & 1 \\
\hline & & & X1d & 1 & 1 \\
\hline & & & Xle & 1 & 4 \\
\hline & & & X1f & 1 & 1 \\
\hline Slb & 1 & 1 & $\mathrm{X} \lg$ & 1 & 2 \\
\hline \multirow[t]{3}{*}{ Slc } & 6 & 2 & Xlh & 5 & 2 \\
\hline & & & $\mathrm{Xli}$ & 1 & 3 \\
\hline & & & $X 1 j$ & 1 & 3 \\
\hline \multirow[t]{4}{*}{ Sld } & 6 & 5 & Xlk & 3 & 5 \\
\hline & & & X11 & 2 & 3 \\
\hline & & & $\mathrm{X} \operatorname{lm}$ & 2 & 3 \\
\hline & & & $\mathrm{X} \ln$ & 6 & 4 \\
\hline Sle & 1 & 4 & $\mathrm{X} 10$ & 1 & 5 \\
\hline Sif & 1 & 6 & $X 1 p$ & 1 & 5 \\
\hline S2 & 1 & 9 & $\mathrm{X} 2$ & 1 & $>9$ \\
\hline
\end{tabular}

The $X b a I$ patterns form subgroups within each SpeI pattern. 
a

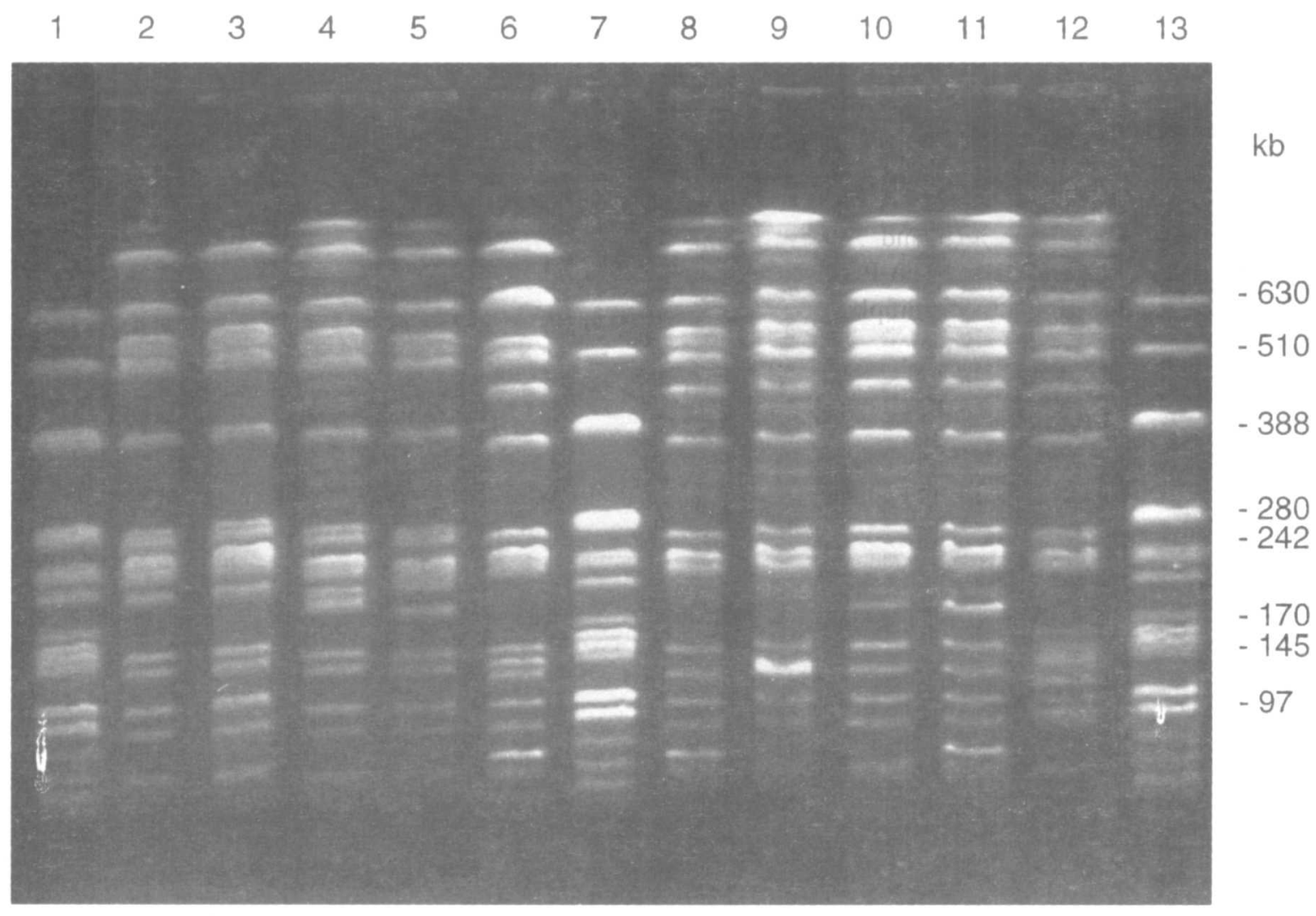

b

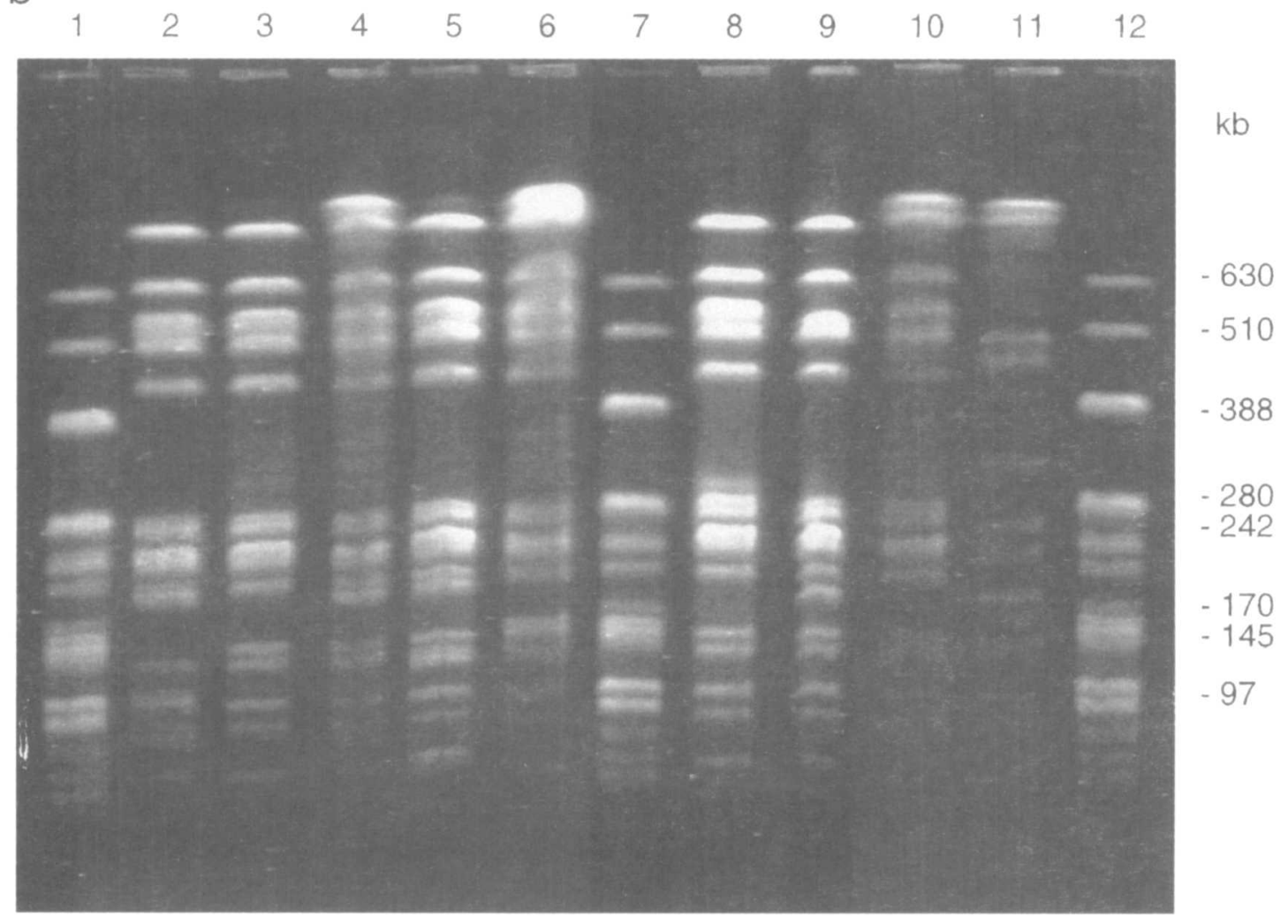

Fig. 1. (a) PFGE patterns produced with restriction enzyme XbaI. Lanes 1, 7 and 13, control strain ATCC $9633 ; 2$ and 3, pattern $X 1 \mathrm{~h} ; 4$, pattern $X 1 j ; 5$, pattern X1i; 6, pattern Xlp; 8, pattern X1k; 9, pattern X1l; 10, pattern X1m; 11, pattern XIn; 12, pattern X1o. (b) PFGE patterns produced with restriction enzyme XbaI. Lanes 1, 7 and 12, control strain ATCC 9633; 2, pattern Xle; 3, pattern X1a; 4 and 5, pattern X1b; 6, pattern X1c; 8, pattern X1d; 9, pattern $\mathrm{X} 1 \mathrm{~g} ;$ 10, pattern X1f; 11, pattern X2. 
(1\%). The patterns were randomly spread in relation to the date of isolation; indeed organisms giving different patterns were isolated on the same day from different patients or sites.

\section{Typing of isolates from patient $D$}

The nine isolates examined from patient $\mathrm{D}$ gave three different $X b a I$ patterns (X1a, X1b and X1c), but all gave the same SpeI pattern (Sla). The PFGE patterns were independent of the date of sampling and two isolates with different $X b a \mathrm{I}$ types were isolated on one occasion from the same specimen. Multiple isolates were examined from seven other patients and, in five of them, more than one PFGE pattern was found.

\section{Discussion}

$K$. pneumoniae is well known for its ability to become resistant to first-line antibiotics and is the bacterial species most likely to produce ESBLs [14]. Many outbreaks of multiresistant klebsiellae have been reported previously [2-9], although most were not as large as this Grampian outbreak. The spread of such organisms causes severe problems for the management of patients. Morbidity and even mortality may increase as a result of resistance to empirical regimens. Costs. escalate if policies are changed to include antibiotics to which multiresistant klebsiellae are susceptible, infection control procedures are implemented and discharges delayed.

It was not possible to test isolates from all 283 patients thought to be involved in the outbreak up to the end of December 1994. Therefore, conclusions were based on a sample of 80 isolates which was chosen to include different aspects of the collection. Isolates were selected to represent all wards in the two hospitals most affected, and to study one ward in detail. As patients move about frequently, however, the ward in which their colonisation was first identified was not necessarily the one where they first became colonised. The fact that ESBL production in $K$. pneumoniae had been detected only in capsular type $\mathrm{K} 2$ isolates in Grampian suggested that this outbreak was due to clonally related organisms. Nevertheless, many unrelated strains can exist within any one capsular type [15], so this evidence was not conclusive.

PFGE indicated that all the test organisms, except one, were closely related whereas they were distinct from local isolates of ESBL-negative $K$. pneumoniae that were susceptible to gentamicin and ciprofloxacin. Therefore, this outbreak appeared to have been caused by the spread of one strain to many people. The one multiresistant isolate found to be unrelated to the outbreak by PFGE was of capsular type K2 and was resistant to gentamicin, but it had other characteristics that distinguished it from the majority of test isolates: it was ESBL-negative, ciprofloxacin-susceptible and from a ward where no other suspected outbreak isolate was ever detected.

Outbreaks due to a single strain have been described where the ESBL gene was carried on the chromosome or a non-transferable plasmid $[2,4,16]$; in others, a single strain was responsible despite the ability of its ESBL gene to transfer at a high frequency in vitro [3]. These last data are in contrast to the dissemination of TEM-3 $\beta$-lactamase in France, where spread was largely due to plasmid transfer among strains or species $[5,6]$. The type of $\beta$-lactamase produced by the Grampian strain, and its ability to transfer to other organisms, are under investigation.

The grouping of isolates obtained after digestion with SpeI correlated well with the XbaI grouping, with three of the six SpeI groups being further subdivided by $X b a \mathrm{I}$. Thus digestion with $X b a \mathrm{I}$ was the more discriminatory method, as has been reported previously for $K$. pneumoniae [9]. The large number of subtypes recorded is likely to be due to the protracted nature of the outbreak, lasting over 3 years, the many patients involved and the large number of isolates characterised. Isolates were most frequently found to be of pattern S1a-X1a. Two possible explanations for the dominance of this genotype are that it is the original genotype and that all others are derived from it or, alternatively, that it has a selective advantage over the other variants. The former hypothesis cannot be tested, as the isolate from the first identified case was not retained and subsequent isolates - which belonged to a variety of different subtypes - date from at least 10 months after the first isolate. The alternative explanation, a selective advantage of the prominent type, is not compatible with the constant proportion of pattern Sla-Xla isolates observed over the duration of the outbreak.

Detailed analysis of nine isolates from a single patient showed that more than one subtype could be present simultaneously without one becoming dominant. In any event, the finding of multiple closely related genotypes is consistent with genetic re-arrangement generating variants which can co-exist.

MICs of antibiotics varied widely among isolates associated with the outbreak but most of its members were resistant to both gentamicin and ciprofloxacin. As resistance to these antibiotics was uncommon in Grampian before the outbreak this is not surprising. However, four isolates susceptible to either gentamicin or ciprofloxacin had the same PFGE pattern as the outbreak strain, whereas one gentamicin-resistant organism was unrelated. In contrast, the absence of ESBL activity was a poor predictor of exclusion from the outbreak: 11 ESBL-negative isolates gave PFGE patterns implicating them in the outbreak. No 
correlation was found between PFGE subtype and antibiotic susceptibility patter. Isolates with identical susceptibility profiles often fell into different PFGE groups and some isolates that were distinguishable on the basis of susceptibility had identical PFGE patterns. The three gentamicin-susceptible isolates gave subtypes Xla, Xlg and Xlj and the imipenem-resistant isolate gave pattern $\mathrm{Xlc}$ and was indistinguishable from 11 other isolates. Furthermore, isolates resistant to gentamicin and ciprofloxacin but without any detectable ESBL activity gave PFGE patterns identical or related to the outbreak strain type. MIC typing would not, therefore, have been a useful method for investigating this outbreak. Without the broad inclusion criteria adopted (i.e., any $K$. pneumoniae with ESBL activity, or resistance to gentamicin, or ciprofloxacin) many outbreak isolates would not have been detected.

This study investigated the molecular epidemiology of one of the largest outbreaks of multiresistant $K$. pneumoniae described in the UK. There is clear evidence that it was due to the spread of a single strain, with no detectable transfer of resistance genes to other $K$. pneumoniae strains. The multiple PFGE subtypes detected do not correlate with any single known marker, including antibiotic susceptibility patterns, and this is under further investigation.

This work was supported by the Scottish Office (grant no. K/MRS/50/ C2368 awarded to F. M. M. and K. J. F. along with I. M. Gould and S. G. B. Amyes). We are grateful to Dr T. L. Pitt and his staff, CPHL, Colindale, London for help with the capsular typing of strains.

\section{References}

1. Hobson RP, MacKenzie FM, Gould IM. An outbreak of multiply-resistant Klebsiella pneumoniae in the Grampian region of Scotland. $J$ Hosp Infect 1996; 33: 249-262.

2. Arlet G, Sanson-le Pors MJ, Rouveau M et al. Outbreak of nosocomial infections due to Klebsiella pneumoniae producing SHV-4 beta-lactamase. Eur J Clin Microbiol Infect Dis 1990; 9: 797-803.

3. Arlet G, Rouveau M, Casin I, Bouvet PJM, Lagrange PH, Philippon A. Molecular epidemiology of Klebsiella pneumo- niae strains that produce SHV-4 $\beta$-lactamase and which were isolated in 14 French hospitals. $J$ Clin Microbiol 1994; 32: 2553-2558.

4. Johnson AP, Weinbren MJ, Ayling-Smith B, Du Bois SK, Amyes SGB, George RC. Outbreak of infection in two UK hospitals caused by a strain of Klebsiella pneumoniae resistant to cefotaxime and ceftazidime. J Hosp Infect 1992; 20: 97103.

5. Brun-Buisson C, Legrand $P$, Philippon A, Montravers F, Ansquer $M$, Duval J. Transferable enzymatic resistance to third-generation cephalosporins during nosocomial outbreak of multiresistant Klebsiella pneumoniae. Lancet 1987; 2: 302306.

6. Kitzis MD, Billot-Klein D, Goldstein FW et al. Dissemination of the novel plasmid-mediated $\beta$-lactamase CTX-1, which confers resistance to broad-spectrum cephalosporins, and its inhibition by $\beta$-lactamase inhibitors. Antimicrob Agents Chemother 1988; 32: 9-14.

7. Bingen EH, Desjardins $\mathrm{P}$, Arlet $\mathrm{G}$ et al. Molecular epidemiology of plasmid spread among extended broad-spectrum $\beta$ lactamase-producing Klebsiella pneumoniae isolates in a pediatric hospital. J Clin Microbiol 1993; 31: 179-184.

8. Poh CL, Yap SC, Yeo M. Pulsed-field gel electrophoresis for differentiation of hospital isolates of Klebsiella pneumoniae. $J$ Hosp Infect 1993; 24: 123-128.

9. Gouby A, Neuwirth C, Bourg G et al. Epidemiological study by pulsed-field gel electrophoresis of an outbreak of extendedspectrum $\beta$-lactamase-producing Klebsiella pneumoniae in a geriatric hospital. J Clin Microbiol 1994; 32: 301-305.

10. National Committee for Clinical Laboratory Standards. Methods for dilution antimicrobial susceptibility test for bacteria that grow aerobically, 2nd edn. Approved Standard. NCCLS Document M7-A2. 1990. Villanova, PA, National Committee for Clinical Laboratory Standards.

11. Jarlier V, Nicolas MH, Fournier G, Philippon A. Extended broad-spectrum $\beta$-lactamases conferring transferable resistance to newer $\beta$-lactam agents in Enterobacteriaceae: hospital prevalence and susceptibility patterns. Rev Infect Dis 1988; 10: $867-878$.

12. Smith CL, Econome JG, Schutt A, Klco S, Cantor CR. A physical map of the Escherichia coli K12 genome. Science 1987; 236: 1448-1453.

13. Tenover FC, Arbeit RD, Goering RV et al. Interpreting chromosomal DNA restriction pattems produced by pulsedfield gel electrophoresis: criteria for bacterial strain typing. $J$ Clin Microbiol 1995; 33: 2233-2239.

14. Jacoby GA, Medeiros AA. More extended-spectrum $\beta$-lactamases. Antimicrob Agents Chemother 1991; 35: 1697-1704.

15. Thompson W, Romance L, Bialkowska-Hobrazanska $\mathrm{H}$, Rennie RP, Ashton F, Nicolle LE. Klebsiella pneumoniae infection on a rehabilitation unit: comparison of epidemiologic typing methods. Infect Control Hosp Epidemiol 1993; 14: 203-210.

16. Jacoby GA, Han P. Detection of extended-spectrum $\beta$ lactamases in clinical isolates of Klebsiella pneumoniae and Escherichia coli. J Clin Microbiol 1996; 34: 908-911. 